\title{
3D Mesh Generation Based on Improved Advancing Front Method
}

\author{
Qianru Xie* and Guohua Geng \\ Institute of Information Science \& Technology, Northwest University, Xian, China \\ ${ }^{*}$ Corresponding author
}

\begin{abstract}
Being able to generate 3D meshes automatically is an important issue in the domain of finite element analysis. A novel algorithm of 3D finite element mesh generation is proposed. The algorithm combines the Octree and advancing front technology to create meshes which have significantly higher quality and lower computational cost than single method. The key step and the main contribution of this work are to apply background grid to control the density and size of mesh, simultaneously reduce the intersection computation costs when internal units are generated. First the background grid is constructed using the Octree, and then the model surface is triangulated according to background grid. After that 3D entity unit is constructed and the whole mesh is optimized finally. The experiments results indicate that, compared with advancing front technology, the increment for its efficiency is about $20 \%$, which reduce the time complexity.
\end{abstract}

Keywords- mesh generation; AFT; Octree

\section{INTRODUCTION}

With finite element analysis seeing increased use in active research areas such as medicine, biology, there is an emerging need for quality mesh generation of the spatially realistic domains that are being studied. The quality of grid division affects the accuracy and velocity of simulation. In recent years, there are some theories and methods of finite element. Several commercial FEM software packages are available and widely used in the practical for industrial engineering design and analysis, such as ABAQUS, ANSYS. The typical approach could be divided into several categories: Octree [1], Delaunay triangulation [2-4] and advancing front technology (AFT) [58].In this paper an fast and accurate approach is proposed, which combine the Octree and AFT method.

\section{RELATED WORKS}

By Octree method, a square grid containing the object to be discretized is recursively subdivided until a desired resolution is reached[1]. But it is insufficient to deal with the boundary problem, so that leads to generate poor quality element on boundary.

In most Delaunay triangulation process, before interior nodes are inserted, a tessellation of the nodes on the domain boundary is produced. However, in this process there is no guarantee that boundary segments will all be present in the triangulation. With the rapid development of the FEM, the Delaunay triangulation algorithm was further extended to generate valid finite element meshes for practical engineering problems [2].
AFT is another mesh generation approach be used widely. The method can locate exact position of new node on curved surface and guarantee high quality elements. When generating new node, some steps should be carried out such as intersection and inclusion between units in order solve the distortion, which will consume considerable computation cost. According to the static the judgment brings about $80 \%$ computation costs, which affects the efficiency of subdivision severely.

Cuillire et al.[5] introduces a analytic expression about the distortion level of mapping by parameter equation of curved surface, then the subdivision is carried out using AFT, finally mapped them onto curved surface to obtain meshes. The algorithm can measure the degree of distortion more accurately, but it doesn't apply to every situation. Lo et al. [6] constructed aided curve to locate the new node by compute the intersection of curve and surface. The approach can obtain high quality meshes aimed the curved surface with Curvature changed slowly. Guan et al. [7] introduces Riemann metric to generate 3D self-adaptation meshes. The method first used Riemann metric to improve AFM, and then build the meshes on parameter domain, mapped the meshes on parameter domain onto curved surface to obtain meshes. It can improve the quality of mesh element, but the implementation is very fussy. Wang et al. [9] etc. constructed orthogonal frame for every node and use mapping algorithm to locate the new node. Lederman et al. [10] employed a grid-based approach to generate initial tetrahedral mesh, and then performed mesh improvement on related material interfaces. The drawback is that it will generate an enormous number of additional elements and nodes. Zhang et al. [11] developed an octreebased isocontouring method with a material change edge analyzing to mesh multi-material image. Due to the inherent drawback of Octree-based method, elements around constraints could not maintain a good shape and quality as required. Xing et al. [12] adopted multilateral marching cubes to generate the background mesh and to remesh the extracted interface meshes by AFT. The flaw is that it will spend more time to execute collision detection within the process of generate tetrahedron. Harmel et al.[13] obtained a volumetric discretization from a Tspline surface representation. The drawback is that the morphing process requires a large number of deformation steps to avoid invalid elements.

Aimed to the problems of large computation and poor convergence of AFT, an improved mesh generation method is presented combined Octree to traditional AFT in this paper. The main idea of this approach is to obtain the background firstly, and then to generate entity mesh, finally to optimize the mesh. The approach lowered intersection and inclusion time of 
AFT, and solve the poor quality problem of boundary elements in Octree.

\section{CONSTRUCTION OF BACKGROUND MESH}

Unit size is difficult to compute directly when generating, so some indirect approach can be taken to determine it. Background grid is such an efficient method. it is special meshes which can involve all units and nodes in specified domain. The element of background is helpful to locate the new unit and compute their size.

In this approach, the background grid is consisted of a set of cubes, which can be used to restrict units size and normal direction. Since Octree can keep the balance of number of cells and approaching precision of boundary, so in this article the Octree method was adopted to obtain created background grid, the level of decomposition of it can control the density of units.

\section{A. Initial Background Grid}

For background grid needs to involve the whole model, the first step is to set up a cube as the root of Octree according to the biggest size of model along the three directions of Cartesian coordinate system. If every surface of the cube is greater than the surface area of model it involved, the cube should be divide into eight sub cubes along the axis, repeat the process until the area of every surface is smaller than that of surface model. In order to explain the procedure more clearly, fig. 1 show it in $2 \mathrm{D}$ ways.

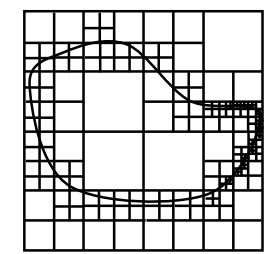

FIGURE I. DIVIDE BACKGROUND GRID

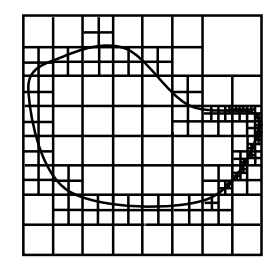

FIGURE II. OPTIMIZE BACKGROUND GRID

Follow above steps, some big Octree node lied in the internal domain decomposition for tetrahedrons inside of model would not be subdivided again, as Fig.1shows. In order to ensure the size of tetrahedrons is uniform, the optimization process should be carried out. To find the biggest node on the surface of model and marks it with Cmax, then use the node traverse the Octree. If the volume of a cube in a node is bigger than Cmax, then the node will be divided until the result is smaller than Cmax. Fig.2 shows the operation result of the process of optimization.

\section{B. Subdivision of Surface Mesh}

It is need to triangulate the whole surface of the model before generating the entity mesh, since the initial wave front is composed by the triangles on model surface. The $2 \mathrm{D}$ version AFT is exploited to accomplish the triangulation. The main step of algorithm is as follows:

a) Obtain the boundary of model surface and discrete it to line segments according to the size decided by background grid. These line segments made up of the set of wave front.

b) Select the line segment with the minimum angle be the initial wave front.

c) According to the direction decided by background mesh, the new node would be insert to compose new unit, and put its edge into the set of wave fronts.

d) Repeat the procedure until all wave fronts participate in the construction of elements. But in the constructing, all the normal vector of triangle mesh should point to the internal model

\section{GENERATION OF ENTITY MESH}

There are many triangles on the model surface after the triangulation. In order to guarantee the smallest triangle can be chosen as initial wave front, the queue should be constructed by the order of size. The treatment can avoid a great deal of units gathered in those smaller areas, which would lead to the quantity reduction of the final mesh.

In order to improve the velocity of subdivision, the hierarchical division methods was adopted. Current wave front queue $Q c$ was set as initial wave front queue $Q$. The next wave front queue was named $Q n$. All the Queues were arranged in the order of size.

\section{A. Compute the Ideal Point}

In order to aid the generation of tetrahedral, the ideal point contributes to determine the location of vertex when the algorithm is executed. First the triangle is taken from the front set as the initial wave front, marked with $\triangle A B C$. The location of ideal point IP can be determined by the centroid of $\triangle A B C$ and the size of background mesh node. Then make a straight line though the centroid of $\triangle A B C$, IP lies in this line, which distance to $\triangle A B C$ is the side length $\mathrm{R}$ of corresponding cube unit in the Octree.

Once the ideal point is computed, the search area can be defined, which is convenience to the location of tetrahedron vertex. The search area is apparent from Fig.3. It is a sphere with the center is IP, the radius is the side length of cube unit. The sphere determined the upper limit of the distance from the target vertex to the centroid $\triangle A B C$. In order to avoid generate narrow and long units, it also demand a lower limit of the distance from the target vertex to the centroid $\triangle A B C$. It is defined as ten percent the distance between the ideal point $I P$ and the centroid $M$. The step not only can enhance the quality of units, but also reduce the search range. 


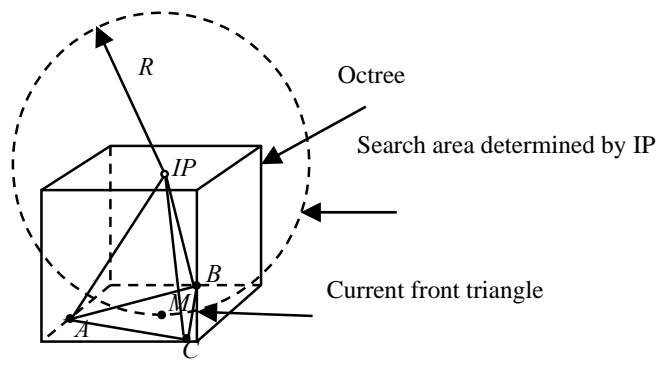

FIGURE III. FIG.3 DETERMINATION OF THE IDEAL POINT

\section{B. Generation of Tetrahedron}

Because of Octree method was adopted in this article, which can control the size of units efficiently, so some tests, such as the check of volume, distance, included angle, need not to execute. Even in intersection tests, Only the test between surfaces need to carry out. For the sake of avoiding such tests, the velocity of generating entity mesh could improve remarkably. The intersection of two triangles could be determined by interval overlap method.

The search queue named $Q s$ is consists of points which is in the current search queue and the sphere meanwhile. The ideal point IP is added at the end of queue $Q s$. The generation of new tetrahedral could be divided in three cases to discuss:

a)if IP is only point contained in $Q s$, the ideal point IP will be as the new vertex, the new tetrahedron consist of by $\triangle A B C$ and it is showed in Fig.3. If the intersection occurred in test, so put $\triangle A B C$ into the queue $Q h$. The queue $Q h$ includes nodes that cannot split temporally.

b)if only one point $\mathrm{P}$ belongs to $Q s$ and $P \in Q c$, the point $P$ will be as the vertex to generate tetrahedron. If the intersection occurred in test, so put $\triangle A B C$ into the queue $Q h$.

c)if multiple point included in $Q s$, and $P i \in Q c, \mathrm{i}=1,2$, maxlength $(Q c)$, the solid angle made up of $\mathrm{Pi}$ and $\triangle A B C$ would be calculated respectively. The result would arrange in order from large to small, then chose a point, which can consist of the biggest solid angle, to be as the vertex of tetrahedron. The purpose is to ensure other search points do not be included in new tetrahedron unit. If the intersection occurred in test, so take other node from $Q s$ and test again

When new tetrahedron was generated, the new wave front is created. The new wave front should be added to the queue $Q n$ the next level wave front, and deleted $\triangle A B C$ from the current wave front queue $Q c$. Then takes next node from $Q c$ as the wave front to compute IP and generate tetrahedral, until $Q c$ is empty. After that nodes in $Q n$ are taken to add to $Q c$, repeat procedure above, until $Q n$ and $Q c$ are all empty, the subdivision process finished.

\section{Subdivision of Remaining Polyhedron}

Firstly is to delete all tetrahedrons which connected with the node in queue $Q h$ to obtain new empty cavity. Next the wave front should be chosen again to generate new unit, the procedure is shown in Fig.4(a), a remaining polyhedron that cannot be divided, because it is not a convex polyhedron. The tetrahedron shows in Fig.4(b) is the part of polyhedron, which is divided before. According to the backtracking algorithm, the tetrahedron should be restored to the original polyhedron to get the empty cavity as shown in Fig.3(c). The re-division result shows in Fig.4(d)

After applying backtracking algorithm repeated, there are still some polyhedron that cannot be divided. If remaining polyhedron is convex polyhedrons, so the liner programming method can be employed to compute the location of insertion point, which will be guarantee every surface to generate effective tetrahedron with the insertion point. But sometimes these remaining polyhedron aren't convex polyhedrons, every surface on them cannot constitute tetrahedron with arbitrary point, such polyhedrons are called Schönhardt polyhedron. The character of it is each vertex has four edge connect to it and there are several edges which normal direction points to inside of the polyhedron.

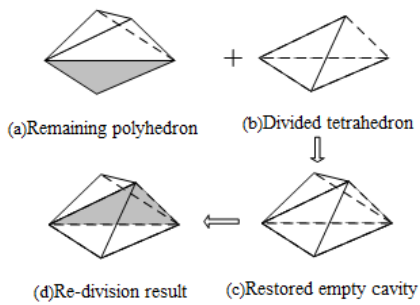

FIGURE IV. RE-DIVISION OF REMAINING POLYHEDRONS

In this case moving vertex method can be applied to solve[14]. The more complex situation is remaining polyhedron is made up of several Schönhardt polyhedron, so the resolution is to divide it into several polyhedrons to handle.

\section{Mesh Optimization}

The mesh optimization must be carry out to eliminate these units to improve the quality of elements. The mesh smoothing method is used here to raise the quality without changing the topology.

The mesh smoothing method is achieved by a weighted Laplace operator. If suppose that a boundary point $\mathrm{P}$ is surrounded by $n$ grid points, then the operator can be described as follows:

$$
L_{p}^{m+1}=L_{p}^{m}+\phi \frac{\sum_{i=1}^{n} \omega\left(L_{i}^{m}-L_{p}^{m}\right)}{\sum_{i=1}^{n} \omega}
$$

The value of the relaxation factor is too large, which leads to the negative volume of the adjusted unit, and thus the topology of the mesh is destroyed. On the contrary, the smaller the value, the smaller the change of the two iteration, which can be stable, which is conducive to maintaining the topology of the grid, but the calculation speed will be affected.

\section{EXPERIMENTS RESULT AND ANALYSIS}

In this experiment, we present applications of our meshing approach to aim to models with different complexity. The first group is cloud models from Stanford model library. The dataset's structure is simple and the amount of data is relatively 
small. Fig.5 showed the division result. The amount faces of modes is from 3,000 to 70,000 . The number of vertex is 5,000 to 10,000 .

Then, the second group subject is based on complex model. The model is the craniofacial model which obtained by reconstruction of CT image. It is more complex, and the amount of data is very big. The number of faces is above 100,000 , and the vertex is the same. Classically the quantity of unit is above 10,000. The experiment result is shown in Fig.6.

In order to estimate the result objectively an quantitatively, the quality factor was introduced to evaluate the result of different method. The quality factor is three times radio of the tetrahedron inradius $r$ and circumradius $R, \beta=3 r / R$.

The higher the value of $\beta$, the higher the quality of the tetrahedral element.Fig.7 is shown the bar graph of quality coefficient distribution.
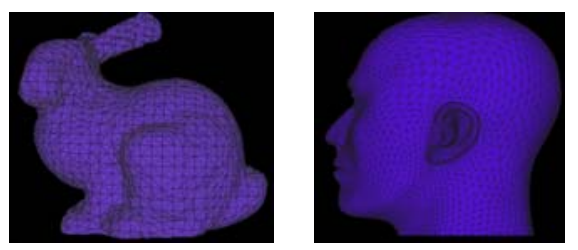

FIGURE V. DIVISION RESULT OF SIMPLE MODEL
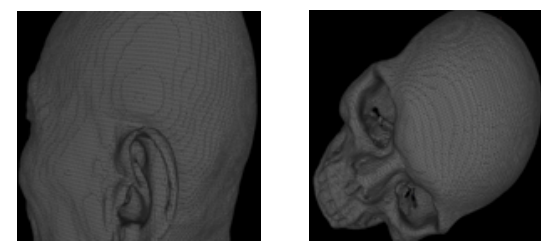

FIGURE VI. DIVISION RESULT OF SKULL MODEL

The amount of elements is the least by using approach in this article when $\beta<0.2$, it's only $0.14 \%$ of the total number of units. The percentage is $1.3 \%$ using the Octree method, to AFT the figure is $0.38 \%$. When $0.8 \leqslant \beta<1.0$, the quantity of units is the most to applying algorithm in this article. Applying Octree method, the figure is $12.9 \%$, to AFT, the percentage is $35.8 \%$.

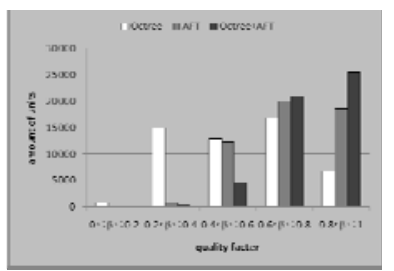

FIGURE VII. COMPARISON OF QUALITY FACTOR

The time complexity of Octree and AFT are all $O(N \log N)$. The test time of including, intersection and distance is above $80 \%$ of total time of execution of the algorithm. In order to prove the efficiency of this algorithm objectively, ten models with different complexity was employed to test triangulation by using AFT and this approach. The execution time of generating mesh by using different method is recorded. The statistic result is shown in Fig.8.
From the result, the velocity improved about $20 \%$ by using this algorithm, compared to AFT. The reason is this method simplified the validity test process such as intersection and inclusion between internal tetrahedral with the aid of Octree, So the technology speed up the mesh generation at same time guarantee the mesh quality. The curve in Fig. 8 shows that the time complexity of this method is approximate to linearity. According the actual velocity, the number of units per minute is sixty thousand or more, which can meet the need of engineering.

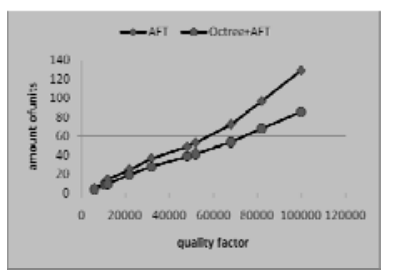

FIGURE VIII. COMPARISON OF THE EXECUTION TIME OF TWO ALGORITHMS

\section{ACKNOWLEDGMENT}

This work was supported by The National Natural Science Foundation of China (61673319) and the Scientific research plan projects of Shaanxi Education Department(2013JK1179)

\section{REFERENCES}

[1] N. Sapidis, R. Perucchio, Combining recursive spatial decomposition and domain Delaunay tetrahedralization for meshing arbitrary shaped solid models, Computer. Methods Application. Mech. Engrg. 108 (1993) 282-302.

[2] H. Borouchaki, S.H. Lo, Fast Delaunay triangulation in three dimensions, Comput. Methods Appl. Mech. Engrg. 128 (1-2) (1995) 153-167.

[3] AHMAD BINIAZ, GHOLAMHOSSEIN. A faster circle-sweep Delaunay triangulation algorithm[J].2012,Vol. 43(1):1-13.

[4] S.H. Lo. Delaunay triangulation of non-uniform point distributions by means of multi-grid insertion[J]. Finite Elements in Analysis and Design.2013,vol.63:8-22

[5] CUILLIRE J C. An adaptive method for the automatic triangulation of 3D parametric surfaces [J] . Computer Aided Design, 1998, 30(2) : 139149

[6] S.H. Lo. Volume discretization into tetrahedra.II. 3D Triangulation by advancing front approach [J]. Computers and Structures, 1991, 39 (5):501-511

[7] GUAN ZH, SUI X,GU Y, Automatic finite element mesh generation over 3D combined surfaces[J].Chinese Journal of Computer Mechanics,2003,20(4):409-416

[8] LAZAROS D. ADAMOUDIS, GERORGIA KOINI, Heuristic repairing operators for $3 \mathrm{D}$ tetrahedral mesh generation using the advancing-front technique. Advances in Engineering Software.2012,54:49-62.

[9] WANG W, FAN H,XI G, A New Algorithm for triangular Mesh Generation[J].Journal of Xi'an Jiao Tong University,2014,48(3):1-7.

[10] Lederman C, Joshi A, Dinov I, Vese L, Toga A, Van Horn JD.. The generation of tetrahedral mesh models for neuroanatomical MRI[J]. NeuroImage 2011,55(1):153-164.

[11] Zhang Y, Hughes TJR, Bajaj CL. 2010. An automatic 3D mesh generation method for domains with multiple materials. Computer Methods in Applied Mechanics and Engineering 199(5-8):405-415.

[12] Huilin Xing and Yan Liu. Mesh Generation for 3D Geological Reservoirs with Arbitrary Stratigraphic Surface Constraints. Procedia Computer Science. Volume 29, 2014, Pages 897-909

[13] Maximilian Harmel, Roger A. Sauer, David Bommes, Volumetric mesh generation from T-spline surface representations[j], Computer-Aided Design.2006 http://dx.doi.org/10.1016/j.cad.2016.07.017 
[14] DU Q,Tezuka Akira,Decomposition of Schönhardt-type Polyhedrons in Advancing front Method[J]. Journal of South China University of technology(Nature Science Edition) 2002,30(8):60-63 